\title{
Three principles for canonical quantum gravity
}

\author{
Rodolfo Gambini ${ }^{1}$, and Jorge Pullin ${ }^{2 *}$ \\ 1. Instituto de Física, Facultad de Ciencias, \\ Iguá 4225, esq. Mataojo, 11400 Montevideo, Uruguay. \\ 2. Department of Physics and Astronomy, \\ Louisiana State University, Baton Rouge, LA 70803-4001
}

(Dated: December 13th, 2012)

\begin{abstract}
We outline three principles that should guide us in the construction of a theory of canonical quantum gravity: 1) diffeomorphism invariance, 2) implementing the proper dynamics and related constraint algebra, 3) local Lorentz invariance. We illustrate each of them with its role in model calculations in loop quantum gravity.
\end{abstract}

* Corresponding author. Email: pullin@lsu.edu. Tel/Fax: +1 2255780464. 


\section{INTRODUCTION}

Since at present we do not have unexplained experimental evidence that requires a quantum theory of gravity for its understanding, we find ourselves in a rather unconventional situation. In physics, theory is usually guided by experiment. The situation is perhaps akin to the one faced by Einstein when developing the general theory of relativity. Although there were some experiments to be explained, he had to be mostly guided by physical principles and intuition. Here we would like to highlight three physical principles that we believe should provide guidance in canonical quantum gravity, and the implications of their use in some model situations.

The first principle is diffeomorphism invariance. No one believes a fundamental theory of gravity should depend on background structures therefore space-time diffeomorphism invariance needs to be implemented. The history of how we ended up with background independence as a principle throughout the history of physics all the way back to the relational ideas of Mach is well recounted by Smolin in [1]. Modern gravity theories are, however, complicated. For instance in general relativity one has several layers of structure to consider. The most elementary is the dimensionality of the space-time. Then its topology. Furthermore there is the differential structure, the signature and finally the metric and fields. We will restrict our discussion to approaches that consider the dimension, differential structure and signature as given (although the introduction of certain measures in Hilbert spaces may imply a change in differential structure, one expects that in semiclassical regimes the differential structure is unchanged). Only diffeomorphism invariant questions about the metric and the fields can be considered physically relevant. Topology change can be accommodated in various approaches to quantum gravity, including the canonical one [2].

Any physical description involves many entities whose properties the theory has the task to describe. The standard description involves some absolute framework with respect to which properties are defined. In Newtonian physics, for instance, the background is a three dimensional Euclidean space and a one dimensional universal time. General relativity essentially is a background independent theory where the fundamental properties of the elementary entities consist entirely of relationships between those elementary entities. In 1912 Einstein had found the basic form of the gravitational field but it took him three years longer to write the equations of motion. His covariance principle required that the laws of 
nature were the same in all reference frames. But in a generally covariant theory statements of the kind of "what is the value of the gravitational field at coordinates $x^{a}$ " make no sense. Indeed, a coordinate transformation can assign a region with large curvature to a coordinate point that prior had low curvature. In 1915 Einstein solved the problem. The idea is that it is only possible to describe relations. For example it is invariant to state that in a region in which certain light rays are present space-time has certain geometric properties (e.g. curvature). Einstein himself put is this way: "the results of our measuring are nothing but verifications of... meetings of the material points of our measuring instrument with other material points, coincidences between the hands of a clock and points on the clock dial and observed point events happening at the same point at the same time." In our view this relational vision of background independence is the main guiding principle that must be followed when constructing a theory of quantum gravity. In such a theory only observable quantities (that are invariant under general coordinate transformations) can be associated with physical quantum operators. In the last few years there has been important progress in the description of the evolution and geometry in terms of such quantities [3].

In the canonical approach, diffeomorphism invariance is reflected in the algebra of constraints. But this is not enough. In particular one has to pay careful attention to modifications that the theory may suffer through the use of non-traditional measures that arises in loop quantum gravity [4]. We will see that this may restrict the types of diffeomorphisms that are recovered in the low energy limit of the theory. The non-traditional measures arise directly as a consequence of diffeomorphism invariance and are fairly unique [5].

Related to the aforementioned principle is the second one: one should properly implement the dynamics of the theory. Since general relativity is a generally covariant theory, the Hamiltonian vanishes and one is just left with a set of constraints from which the dynamics needs to be disentangled. The constraints satisfy an algebra that needs to be implemented at a quantum level. Enforcing the constraint algebra assures that the canonical framework, which splits space-time into space and time, represents a space-time diffeomorphism invariant theory [6]. This poses tight constraints on the quantization process that otherwise contains a large degree of ambiguity. In particular if one uses lattices to regularize the theory, reproducing the algebra of constraints can become quite a challenge.

The last principle is local Lorentz invariance. What is meant by this in the context of canonical quantum gravity is that if one studies the low energy limit, the resulting graviton 
(and other particles if one couples the theory to matter) should have propagators that deviate from Lorentz invariance at most only slightly. We will illustrate with a calculation what is meant by "slightly" in this context. In particular, deviations from Lorentz invariance that become large at the Planck scale are unacceptable as was argued by Collins et. al. [7].

We will provide examples of the three principles in action in the following sections.

\section{DIFFEOMORPHISM INVARIANCE}

The first guiding principle is diffeomorphism invariance, or to put it in other terms, background independence. Most physicists believe a modern theory of gravity should not depend on background structures, since then one would have to motivate where the structures came from, and the whole point of general relativity was to eliminate any preferred observers in nature.

In canonical gravity one uses a $3+1$ dimensional split to formulate the equations of the theory. That split, obviously, violates space-time diffeomorphism invariance. The resulting framework is still invariant under spatial diffeomorphisms, such symmetry being reflected in the presence of the diffeomorphism constraint. Spatial diffeomorphism invariance plays a key role in loop quantum gravity. It essentially determines the kinematical structure of the theory through the selection of an inner product that is unconventional from the point of view of ordinary field theories [5]. In turn, this structure implies that physical operators, like those representing areas and volumes, have discrete spectra [8].

The breakage of space-time diffeomorphisms only means that the equations are not invariant, the resulting theory still is. In fact, the algebra of constraints is known to enforce that the resulting formalism is space-time diffeomorphism invariant [6]. So, in principle, if upon quantization one ended up with a set of operators representing constraints that under commutators close an algebra isomorphic to the classical one under Poisson brackets, one could be confident that the resulting quantum theory is space-time diffeomorphism invariant.

But as we mentioned, one faces difficulties in implementing the constraint algebra at a quantum level. Up to present, no models have met such requirement (loop quantum cosmology, where there are no spatial degrees of freedom, implements them trivially so it is really not a strong guiding principle for those models). Moreover, it is customary to propose to deal with the diffeomorphism and Hamiltonian constraints separately. The 
diffeomorphism constraint is solved via the group averaging technique [9], a procedure that cannot be implemented for the Hamiltonian constraint. Treating the constraints differently raises the possibility that space-time diffeomorphism invariance will be violated.

One way to deal with the problem is to gauge fix the theory, eliminating some or all the constraints. Classically, a gauge fixed theory is by definition diffeomorphism invariant. Although it is not manifestly diffeomorphism invariant, since one is dealing with the theory in a form that has no gauge symmetries, the results are diffeomorphism invariant in the sense that they can later be translated into any gauge in terms of gauge dependent variables.

But upon quantization, even in gauge fixed scenarios, there are subtleties. For instance, it can happen that the resulting variables that appear in the models have different ranges of values than those in the classical theory. That can imply that the set of diffeomorphisms considered is a restricted one.

An example of this is present in the treatment of the exterior of a vacuum black hole space-time we discussed in [10]. In that case, one can gauge fix the variables to spherical symmetry. One is left with two canonical pairs, one "longitudinal" along the radial direction $E^{x}, A_{x}$ and a "transverse" one $E^{\varphi}, A_{\varphi}$, with the variables depending on the radial coordinate $x$ and time $t$. One can further gauge fix the radial variable so that the diffeomorphism constraint is gone. The resulting Hamiltonian constraint is

$$
\begin{aligned}
H= & -\frac{E^{\varphi}}{(x+a) \gamma^{2}}\left(\frac{A_{\varphi}^{2}(x+a)}{8}\right)^{\prime}-\frac{E^{\varphi}}{2(x+a)} \\
& +\frac{3(x+a)}{2 E^{\varphi}}+(x+a)^{2}\left(\frac{1}{E^{\varphi}}\right)^{\prime}=0,
\end{aligned}
$$

where $a$ is a constant and $\gamma$ is the Barbero-Immirzi parameter. Multiplying by $\frac{2(x+a)}{E^{\varphi}}$ and grouping terms as,

$$
H=\left(\frac{(x+a)^{3}}{\left(E^{\varphi}\right)^{2}}\right)^{\prime}-1-\frac{1}{4 \gamma^{2}}\left((x+a) A_{\varphi}^{2}\right)^{\prime}=0,
$$

yields an Abelian constraint. Since the constraint is a total derivative, it can immediately be integrated to yield,

$$
\int H d x=C=\left(\frac{(x+a)^{3}}{\left(E^{\varphi}\right)^{2}}\right)-x-\frac{1}{4 \gamma^{2}}\left((x+a) A_{\varphi}^{2}\right),
$$

with $C$ a constant of integration. At $x=0$ one can impose isolated horizon boundary conditions, which imply $1 / E^{\varphi}=0$ and $A_{\varphi}=0$, and this implies that the constant of 
integration $C$ vanishes. Imposing that the metric at infinity asymptotically approach the Schwarzschild solution, which in these coordinates means that, $E^{\varphi}=x+3 M, A_{\varphi}=0$, one concludes that $a=2 M$.

In order to quantize this model, one can discretize the radial variable, and one is essentially left with a system that is "a loop quantum cosmology at every point" and one can borrow the techniques used for cosmology to quantize. The discretized Hamiltonian reads,

$$
\begin{aligned}
H_{m}^{\rho}= & \frac{1}{\epsilon}\left[\left(\frac{\left(x_{m}+2 M\right)^{3} \epsilon^{2}}{\left(E_{m}^{\varphi}\right)^{2}}-\frac{\left(x_{m-1}+2 M\right)^{3} \epsilon^{2}}{\left(E_{m-1}^{\varphi}\right)^{2}}\right)-\epsilon\right. \\
& -\frac{1}{4 \gamma^{2} \rho^{2}}\left(\left(x_{m}+2 M\right) \sin ^{2}\left(\rho A_{\varphi, m}\right)\right. \\
& \left.\left.-\left(x_{m-1}+2 M\right) \sin ^{2}\left(\rho A_{\varphi, m-1}\right)\right)\right],
\end{aligned}
$$

expression that recovers (2) in the limit $\epsilon \rightarrow 0, \rho \rightarrow 0$. In the above expression $x_{m}$ are the positions of the lattice points and $\epsilon$ is the separation of two points in a fiducial metric. As in loop quantum cosmology, we have polymerized the variable $A_{\varphi}$ and $\rho$ is the polymerization parameter. One can show that the discrete constraint constructed is still Abelian and proceed to quantize. The details are in [10].

One can actually solve the constraint and find the physical space of states and recover the quantization that Kuchař [11] had carried out for the same system using metric variables. There is only one degree of freedom, given by $M$ and the wavefunctions are functions of $M$.

To study diffeomorphism invariance, one can reconstruct the non-trivial components of the metric as evolving constants of the motion that are functions of $M$ and a free parameter $A_{\varphi}$. Their explicit form is given by,

$$
\begin{aligned}
& g_{00}=-1+\frac{2 M}{x+2 M} \\
& g_{0 x}=\frac{A_{\varphi}}{2 \gamma \sqrt{1-\frac{2 M}{x+2 M}+\frac{A_{\varphi}^{2}}{4 \gamma^{2}}}} \\
& g_{x x}=\frac{1}{1-\frac{2 M}{x+2 M}+\frac{A_{\varphi}^{2}}{4 \gamma^{2}}} .
\end{aligned}
$$

These are Dirac observables that are functions of a parameter, as is usual for evolving constants of the motion.

The variable $A_{\varphi}$ is free and represents the space-time diffeomorphism freedom left. For instance, for $A_{\varphi}=0$ one has the usual Schwarzschild coordinates and the metric is diagonal. For non-vanishing values one is considering a non-comoving system of coordinates. The 
same expressions can be recovered as quantum operators. In that case one has

$$
\begin{aligned}
& g_{00}=-1+\frac{2 M}{x+2 M} \\
& g_{0 x}=\frac{\sin \left(\rho A_{\varphi}\right)}{2 \rho \gamma \sqrt{1-\frac{2 M}{x+2 M}+\frac{\sin \left(\rho A_{\varphi}\right)^{2}}{4 \rho^{2} \gamma^{2}}}} \\
& g_{x x}=\frac{1}{1-\frac{2 M}{x+2 M}+\frac{\sin \left(\rho A_{\varphi}\right)^{2}}{4 \rho^{2} \gamma^{2}}} .
\end{aligned}
$$

and we see that due to the polymer nature of the representation used, one has the sine functions appearing in places where $A_{\varphi}$ appeared before. Again $A_{\varphi}$ is a free parameter, but we now see that we are not recovering all the possible coordinate systems we had in the classical case due to the finite range of the sine function. The quantum theory therefore has a restricted set of symmetries with respect to the classical theory.

The example is too simple to draw too many conclusions from the observed behavior.One could for instance argue that in quantum gravity it would not be natural to consider diffeomorphisms that blow up regions of sub-Planck scale to ordinary scales. Unfortunately this model is just too simple to conclude anything on that point. But it serves as a warning that there can be additional subtle issues when one enforces diffeomorphism invariance at the quantum level.

\section{THE DYNAMICS AND THE CONSTRAINT ALGEBRA}

General relativity is a generally covariant theory. As such, the Hamiltonian vanishes and the total Hamiltonian is a combination of constraints. If one uses Ashtekar's variables, given by a set of densitized triads $\tilde{E}_{i}^{a}$ and connections $A_{a}^{i}$, one has that the constraints are [12],

$$
\begin{aligned}
\mathcal{G}(\lambda) & =\int d^{3} x \lambda^{i} D_{a} \tilde{E}_{i}^{a}=0 \\
C(\vec{N}) & =\int d^{3} x N^{b}\left[\tilde{E}_{i}^{a} F_{a b}^{i}-A_{a}^{i} D_{b} \tilde{E}_{i}^{a}\right]=0 \\
H(M) & =\int d^{3} x M\left[\epsilon_{i j k} \tilde{E}_{i}^{a} \tilde{E}_{j}^{b} F_{a b}^{k}+2 \frac{\left(\gamma^{2}+1\right)}{\gamma^{2}}\left(\tilde{E}_{i}^{a} \tilde{E}_{j}^{b}-\tilde{E}_{j}^{a} \tilde{E}_{i}^{b}\right)\left(A_{a}^{i}-\Gamma_{a}^{i}\right)\left(A_{b}^{j}-\Gamma_{b}^{j}\right)\right]=
\end{aligned}
$$

They are known, respectively as the Gauss law $G(\lambda)$, diffeomorphism constraint $C(\vec{N})$ and the Hamiltonian constraint $H(M)$. We have presented them smeared with arbitrary test functions $\lambda^{i}, N^{a}, M$ since it makes cleaner the computations of the constraint algebra. As 
before, the parameter $\gamma$ is the Barbero-Immirzi parameter. Different values of the parameter represent the same classical theory, expressed in different variables. These constraints satisfy the algebra,

$$
\begin{aligned}
\{G(\lambda), G(\mu)\} & =G([\lambda, \mu]) \\
\{C(\vec{N}), C(\vec{M})\} & =C\left(\mathcal{L}_{\vec{N}} \vec{M}\right), \\
\{C(\vec{N}), G(\lambda)\} & =G\left(\mathcal{L}_{\vec{N}} \lambda\right), \\
\{C(\vec{N}), H(M)\} & =H\left(\mathcal{L}_{\vec{N}} M\right), \\
\{H(N), H(M)\} & =C(\vec{K})
\end{aligned}
$$

where $K^{a}=\tilde{E}_{i}^{a} \tilde{E}^{b i}\left(N \partial_{b} M-M \partial_{b} N\right) /(\operatorname{det}(q))$.

Notice that the vector $K^{a}$ is not just a combination of derivatives of test functions but actually involves the canonical variables. That means that although the Poisson bracket of two Hamiltonian constraints is proportional to a diffeomorphism, the proportionality factor depends on the canonical variables. This is unlike any of the other Poisson brackets. Since upon quantization the canonical variables get promoted to operators, to ensure the proportionality of the Poisson brackets to a combination of constraints will become problematic. In fact, it is known that if one promotes the constraints to self-adjoint operators, there does not exist a factor ordering that is compatible with the constraint algebra. This means that de facto the Dirac quantization procedure as originally envisioned cannot treat this type of system [13]. Extensions are needed. Two of such extensions are the master constraint program of Thiemann and collaborators [12] and the closely related uniform discretization approach [14] that we have been developing, based on discretizing the time evolution.

The idea of the master constraint is as follows: consider the unsmeared version of the Hamiltonian constraint we introduced above (eliminate the spatial integral and the test function $\mathrm{M}$, and end up with a function of point $\tilde{H}(x))$. One then constructs the master constraint,

$$
M=\frac{1}{2} \int d^{3} x \frac{\tilde{H}^{2}(x)}{\sqrt{\operatorname{det}(q)}} .
$$

Notice that this is only one constraint whereas $\tilde{H}(x)$ were infinitely many. It is clear that if $M$ vanishes so do the infinitely many $\tilde{H}(x)^{\prime}$ 's. One may ask if it is legitimate to claim that the two pictures are equivalent, at least at the classical level. For instance, consider the Poisson bracket of the master constraint with any quantity. Since the master constraint 
is quadratic in the constraint, when you compute its Poisson bracket with any quantity, the result is proportional to the constraint, therefore it vanishes when constraints are enforced. So it seems that the notion of observable is lost. But if you consider

$$
\{\{M, O\}, O\}=0
$$

this condition is equivalent to $O$ being a Dirac observable. So the master constraint can capture the information about observables.

The master constraint is diffeomorphism invariant (and $s u(2)$ invariant as well). And being a single constraint it commutes with itself. So if one considers the master constraint together with the diffeomorphism constraint, they have a very simple constraint algebra,

$$
\begin{array}{r}
\{C(\vec{N}), M\}=0, \\
\{M, M\}=0
\end{array}
$$

and the usual algebra between diffeomorphisms. This is a huge advantage at the time of quantization. The task is to promote the master constraint to a quantum operator and to find the quantum states that are annihilated by it. The advantage is that since the master constraint is a diffeomorphism invariant quantity, there is no doubt that it can be promoted to an operator on the space of diffeomorphism invariant states. And the issue of the structure functions in the algebra of constraints is bypassed. The resulting quantization will not necessarily be equivalent to a canonical quantization in all cases. So this can be seen as a generalization of Dirac's canonical quantization procedure.

The only caveat is, what happens if one discovers that as a quantum operator the master constraint does not have zero among its eigenvalues? In that case the proposal is to consider the smallest eigenvalue. One would not be dealing with a theory where the constraints are enforced exactly but with a theory where the constraints are small ${ }^{1}$. Therefore the theory will not have the same exact symmetries as the classical theory one started with but will have symmetries that approximate those of the classical theory. On the other hand, getting zero as an eigenvalue for the master constraint will be a guideline to deal with the types of ambiguities that one faces when discussing the Hamiltonian constraint.

\footnotetext{
${ }^{1}$ Dittrich and Thiemann propose subtracting the minimum eigenvalue. Even in that case, in the models studied, the quantization does not completely agree with the refined algebraic quantization method [15]
} 
But one need not limit oneself to using a master constraint constructed only with the Hamiltonian constraint. One could in principle build a master constraint by considering the "sum of squares" of all constraints. That would help treating all of the constraints on the same footing. It might surprise the reader that one would attempt to treat the diffeomorphism constraint in this way, but it actually can be done. We have shown in it a simple model, the $1+1$ dimensional version of the Kuchař-Husain [16] model. There the only constraint is the diffeomorphism constraint. We applied the uniform discretization approach.

The uniform discretization approach [14] is based on discretizing the theory and constructing the master constraint and using the considerable freedom one has when discretizing a theory to cast the evolution equations into a form in which evolution is generated by the master constraint,

$$
A_{n+1}=A_{n}+\left\{A_{n}, M\right\}+\left\{\left\{A_{n}, M\right\}, M\right\}+\cdots
$$

where $A$ is any of the canonical variables of the theory. We have shown that this evolution corresponds to the one generated by Hamilton's principal function of the continuum theory while taking a discrete time step. This idea has been further developed by Bahr and Dittrich into the notion of "perfect action" [17].

The beauty of this particular form of the evolution equations is that the value of the master constraint $M$ is preserved exactly. So if one starts with a small value (meaning that one is close to the continuum theory where the master constraint vanishes), one remains close to the continuum theory upon evolution. Suppose we choose that small value to be $\delta / 2$ and let us say we are dealing with a theory with $N$ constraints $\phi^{i}(q, p)=0$. If you define $\lambda_{i}=\phi_{i} / \delta$ (which means $\sum_{i=1}^{N} \lambda_{i}^{2}=1$ ) then the evolution of one of the dynamical variables, say, $q$ can be expanded in $\delta$ and one gets,

$$
q_{n+1}=q_{n}+\sum_{i=1}^{N}\left\{q_{n}, \phi_{i}\right\} \lambda_{i} \delta+O\left(\delta^{2}\right)
$$

and we recognize in the second term the usual evolution one would get with a total Hamiltonian $H_{T}=\sum_{i=1}^{N} \lambda_{i} \phi_{i}$. So we are getting to leading order the traditional evolution equation for a totally constrained system like the ones we discussed in Chapter 4, only discretized. The "step" in the evolution is controlled by the value of $\delta$ and we choose that value by picking initial data such that the master constraint evaluated on them is $\delta / 2$. So we see we have complete control over the approximation. 
We tested these ideas in a version of the $1+1$ dimensional Kuchař-Husain model [18]. The model consists of considering spherically symmetric gravity and ignoring the Hamiltonian constraint. One only has the diffeomorphism constraint. There are two pairs of canonical variables, one "radial" $E^{x}, K_{x}$ (x is the radial coordinate) ${ }^{2}$ and "transverse" $E^{\varphi}, K_{\varphi}$. The diffeomorphism constraint reads $\phi=-\left(E^{x}\right)^{\prime} K_{x}+E^{\varphi} K_{\varphi}^{\prime}$. One builds the master constraint out of it, like one would do for a set of constraints $\phi_{a}$,

$$
\mathbb{H}=\frac{1}{2} \int d x \phi_{a} \phi_{b} \frac{g^{a b}}{\sqrt{g}}
$$

which motivates in our example to choose,

$$
\mathbb{H}=\frac{1}{2} \int d x \phi \phi \frac{\sqrt{E^{x}}}{\left(E^{\varphi}\right)^{3}} .
$$

The quantum states of the theory are given by the direct product of the "point holonomies" for the variables $K_{\varphi}$ and $K_{x}$, and denoted graphically as,

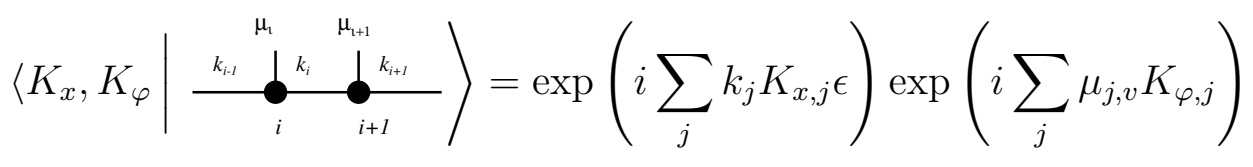

where $k$ and $\mu$ are polymerization parameters (the parameters that appear in point holonomies), $\epsilon$ as before the lattice spacing and the sums go through all the points on the lattice. For simplicity we consider a finite lattice ignoring at the moment boundary issues.

A detailed calculation [18] shows that if one considers a normalized state obtained by superposing all possible states with a given insertion (such a state would be the analogue in the discrete theory of a "group averaged" state)

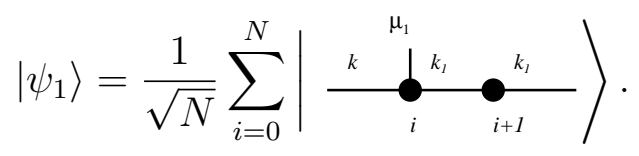

one can show that

$$
\hat{\mathbb{H}}\left|\psi_{1}\right\rangle=0
$$

One can show that similar results hold for larger number of insertions. The master constraint does not vanish but the contributions go as $O(1 / N)$ and therefore if one takes the continuum ${ }^{2}$ We changed notation from section 2 , using $K_{x}$ instead of $A_{x}$ and $K_{\varphi}$ instead of $A_{\varphi}$ in order to be
compatible with the published literature. 
limit they vanish. It is quite remarkable that from such a different picture group averaging arises. Again this is because in order to implement the (in this case very limited) dynamics one has to choose the discretization carefully and that limits significantly what one can do. And it also shows that the master constraint need not be zero if one is not considering the continuum limit. In that case one does not have diffeomorphism invariance, but the closest thing to it on a lattice.

\section{LOCAL LORENTZ INVARIANCE}

Local Lorentz invariance (LLI) has been established with enormous accuracy through particle physics experiments. This makes any deviation from it very problematic for a theory. It creates severe constraints for theories that may include elements that break LLI, for instance, invoking a fundamental lattice structure for space-time. Loop quantum gravity does not automatically violate Lorentz invariance. Some have argued that the presence of a discrete minimum non-vanishing value for the area operator may cause problems but this has been debunked [19]. Essentially the situation is similar to that of angular momentum in quantum mechanics. The fact that its eigenvalues can only take discrete values does not mean that rotational invariance is broken. However, some proposals (in loop quantum gravity and other approaches) include the use of a lattice regularization in which the limit of the lattice spacing going to zero, like one considers in lattice QCD, is not taken. A finite Planckscale lattice remains. That could lead to breakage of Lorentz invariance. When one takes the low energy limit one will end up with propagators that are not Lorentz invariant. There have been a lot of explorations of possible forms of violation of LLI from a phenomenological point of view (see for instance [20]), up to now with no positive experimental evidence.

Collins et al. [7] have studied a model in which one considers a propagator

$$
G(k, m)=\frac{1}{m^{2}+k^{2}+f\left(k / k_{\text {Planck }}\right)+k_{0}^{2}}
$$

where $k_{\text {Planck }}$ is the Planck momentum and we are consider Euclidean $1+1$ dimensions for simplicity. $f(x)$ is a function such that $f(0)=0$ that represents deviations from LLI. One may think, since one has $k / k_{\text {Planck }}$ in it that this will not contribute significantly at low energies and therefore will avoid the experimental constraints. But this is incorrect. In perturbative quantum field theory the propagator appears in loops, and there it is integrated 
in momentum from zero to a cutoff, that in this case we can take to be the Planck scale. In those integrals the propagator deviates importantly from LLI and this leads to unacceptable deviations in experimental predictions.

Does such a propagator emerge in the low energy limit of loop quantum gravity? Currently we do not know. We have argued that perhaps not [21]. For instance, if one considers quantum field theory on a lattice the LLI violating type of propagator that arises is given by

$$
G(k, m)=\frac{1}{m^{2}+a^{-2} \sum_{j=1}^{3}\left(2-2 \cos ^{2}\left(a k_{j}\right)\right)+(b a)^{-2}\left(2-2 \cos ^{2}\left(b a k_{0}\right)\right)},
$$

with $a, a b$ the lattice spacings in space and time respectively. The presence of the trigonometric functions breaks LLI but implies that in the integrals that appear in loop calculations the deviations are small (provided $b$ is close to one). Should a propagator like that emerge in the low energy limit, one could have violations of LLI that are acceptable experimentally.

This example has been criticized [22] in that by working in Euclidean space one introduces an additional symmetry that is not there in the Lorentzian case, in which the contributions would still be large. Other examples can be presented, for instance based on supersymmetry [23] or a modified Pauli-Villars regularization [21] that also lead to propagators that do not introduce large contributions. The jury is still out about what is the situation in loop quantum gravity. But this (the contact with perturbative quantum field theory) is a point that loop quantum gravity at some point will have to address.

\section{SUMMARY}

We have argued that local Lorentz invariance, enforcement of the constraint algebra and diffeomorphism invariance are three basic principles that one should have in a canonical quantization of gravity and illustrated in all cases the implications of enforcing them. Loop quantum gravity is barely starting to deal with models where these issues can be probed in full. We expect in the next few years that we will see these principles playing a stronger guiding role in the constructions of quantum gravity models of increasing complexity. 


\section{ACKNOWLEDGMENTS}

This work was supported in part by grant NSF-PHY-0650715, funds of the Hearne Institute for Theoretical Physics, CCT-LSU and Pedeciba. This publication was made possible through the support of a grant from the John Templeton Foundation. The opinions expressed in this publication are those of the author(s) and do not necessarily reflect the views of the John Templeton Foundation.

[1] L. Smolin, In *Rickles, D. (ed.) et al.: The structural foundations of quantum gravity* 196-239 [hep-th/0507235].

[2] G. T. Horowitz, Class. Quant. Grav. 8, 587 (1991).

[3] R. Gambini, R. A. Porto, J. Pullin and S. Torterolo, Phys. Rev. D 79, 041501 (2009) [arXiv:0809.4235 [gr-qc]].

[4] Ashtekar, A. and Lewandowski, J. (1997) Class. Quan. Grav. 14, A55.

[5] Lewandowski, J., Okołów, A., Sahlmann, H. and Thiemann, T. (2006). Commun. Math. Phys. 267, 703; Fleischhack, C. (2006). Phys. Rev. Lett. 97, 061302.

[6] C. Teitelboim, Ann. Phys. 79, 542 (1973); S.A. Hojman, K.V. Kuchař and C. Teitelboim, Ann. Phys. 96, 88 (1976).

[7] Collins, J., Perez, A., Sudarsky, D., Urrutia, L. and Vucetich, J. (2004). Phys. Rev. Lett. 93, 191301.

[8] Rovelli, C. and Smolin, L. (1995). Phys. Rev. D 52, 5743.

[9] A. Ashtekar, J. Lewandowski, D. Marolf, J. Mourao and T. Thiemann, J. Math. Phys. 36, 6456 (1995) [gr-qc/9504018]; D. Giulini and D. Marolf, Class. Quant. Grav. 16, 2489 (1999) [gr-qc/ 9902045].

[10] R. Gambini and J. Pullin, Adv. Sci. Lett. 2, 251 (2009) [arXiv:0807.4748 [gr-qc]].

[11] K. V. Kuchar, Phys. Rev. D 503961 (1994) [gr-qc/9403003].

[12] Thiemann, T. (2008). Modern canonical quantum general relativity. Cambridge University Press, Cambridge.

[13] K. V. Kuchar, Int. J. Mod. Phys. Proc. Suppl. D 20, 3 (2011).

[14] Campiglia, M., Di Bartolo, C., Gambini, R. and Pullin, J. (2006). Phys. Rev. D74, 124012. 
[15] B. Dittrich and T. Thiemann, Class. Quant. Grav. 23, 1089 (2006) [gr-qc/0411140].

[16] V. Husain and k. V. Kuchar, Phys. Rev. D 42, 4070 (1990).

[17] B. Bahr and B. Dittrich, Phys. Rev. D 80, 124030 (2009) [arXiv:0907.4323 [gr-qc]].

[18] R. Gambini and J. Pullin, Class. Quant. Grav. 26, 035002 (2009) [arXiv:0807.2808 [gr-qc]].

[19] C. Rovelli and S. Speziale, Phys. Rev. D 67, 064019 (2003) [gr-qc/0205108].

[20] Lect. Notes Phys. 541, 1 (2000) [gr-qc/9910089]

[21] R. Gambini, S. Rastgoo and J. Pullin, Class. Quant. Grav. 28, 155005 (2011) [arXiv:1106.1417 [gr-qc]].

[22] J. Polchinski, Class. Quant. Grav. 29, 088001 (2012) [arXiv:1106.6346 [gr-qc]].

[23] S. Groot Nibbelink and M. Pospelov, Phys. Rev. Lett. 94, 081601 (2005) [hep-ph/0404271]. 